\title{
Reliability of ambulatory walking activity in patients with hematologic malignancies
}

\author{
Knols, R H ; de Bruin, E D ; Aufdemkampe, G ; Uebelhart, D ; Aaronson, N K
}

\begin{abstract}
OBJECTIVES: To determine the relative and absolute reliability of the assessment of ambulatory walking activity during 2 consecutive weeks in patients with hematologic malignancies recovering at home from their medical treatment and to compare the physical activity level of hematologic cancer patients after high-dose chemotherapy with healthy subjects. DESIGN: Test-retest study of 2 consecutive 7-day recordings using the microprocessor-based step accelerometer 3 (SAM3). SETTING: Home and community. PARTICIPANTS: Patients $(\mathrm{n}=23)$ with hematologic malignancies recovering from high-dose chemotherapy and healthy controls $(n=30)$. INTERVENTIONS: Not applicable. MAIN OUTCOME MEASURES: The intraclass correlation coefficient $(\operatorname{ICC}(3,1))$ and its $95 \%$ confidence interval (CI), SE of measurement procedure and its $95 \% \mathrm{CI}$, the smallest detectable difference (SDD), the coefficient of variation $(\mathrm{CV})$, and $\mathrm{t}$ tests for the variables total steps and peak activity. RESULTS: The day-to-day and week-to-week CVs for walking activity and peak activity were $35.17 \%$ and $13.17 \%$ and $18.61 \%$ and $6.90 \%$, respectively. For relative reliability, the ICCs for 2 consecutive 7 -day recordings including the 95\% CI for total steps and peak activity were 0.90 (95\% CI, 0.75-0.98) and 0.85 (95\% CI, 0.66-0.94), respectively. The absolute reliability for total steps and peak activity including the SE of measurement procedure and the $95 \%$ CI were $564(95 \% \mathrm{CI},+/-1106)$ and 2.42 steps $(95 \% \mathrm{CI},+/-4.74)$, respectively, for 2 consecutive 7-day recordings. The week-to-week SDD was 1564 for total steps and 6.70 for peak activity. The 7-day mean for total step activity was 5355 for the patients with hematologic malignancies and 6364 for healthy subjects $(\mathrm{P}<0.05)$. CONCLUSIONS: The results of this study indicate that there is good relative reliability for the assessment of 2 consecutive 7 -day recordings of ambulatory walking activity, and it showed that the SDD derived from this sample may be useful in detecting changes in daily walking activity in hematologic cancer patients who are recovering from intensive medical treatment. The study also documented compromised levels of ambulatory walking activity among hematologic cancer patients recovering from high-dose chemotherapy as compared with healthy controls.
\end{abstract}

DOI: https://doi.org/10.1016/j.apmr.2008.06.020

Posted at the Zurich Open Repository and Archive, University of Zurich

ZORA URL: https://doi.org/10.5167/uzh-12457

Journal Article

Accepted Version

Originally published at:

Knols, R H; de Bruin, E D; Aufdemkampe, G; Uebelhart, D; Aaronson, N K (2009). Reliability of ambulatory walking activity in patients with hematologic malignancies. Archives of Physical Medicine and Rehabilitation, 90(1):58-65.

DOI: https://doi.org/10.1016/j.apmr.2008.06.020 
Reliability of ambulatory walking activity in patients with hematologic malignancies

Ruud Knols, Eling D. de Bruin, Geert Aufdemkampe, Daniel Uebelhart, Neil K. Aaronson

Arch Phys Med Rehabil 90: 58-65 @ 2009 American Congress of Rehabilitation Medicine 
Objectives To determine the relative and absolute reliability of the assessment of ambulatory walking activity during 2 consecutive weeks in patients with hematologic malignancies recovering at home from their medical treatment and to compare the physical activity level of hematologic cancer patients after high-dose chemotherapy with healthy subjects. Design Testretest study of 2 consecutive 7-day recordings using the microprocessor based step accelerometer 3 (SAM3). Setting Home and community. Participants Patients $(n=23)$ with hematologic malignancies recovering from high-dose chemotherapy and healthy controls $(n=30)$. Interventions Not applicable. Main Outcome Measures The intraclass correlation coefficient (ICC3,1) and its $95 \%$ confidence interval $(\mathrm{Cl})$, SE of measurement procedure and its $95 \% \mathrm{Cl}$, the smallest detectable difference (SDD), the coefficient of variation $(\mathrm{CV})$, and $t$ tests for the variables total steps and peak activity. Results The day-to-day and week-to-week CVs for walking activity and peak activity were $35.17 \%$ and $13.17 \%$ and $18.61 \%$ and $6.90 \%$, respectively. For relative reliability, the ICCs for 2 consecutive 7-day recordings including the $95 \% \mathrm{Cl}$ for total steps and peak activity were $0.90(95 \% \mathrm{Cl}, 0.75-0.98)$ and $0.85(95 \% \mathrm{Cl}, 0.66-0.94)$, respectively. The absolute reliability for total steps and peak activity including the SE of measurement procedure and the $95 \% \mathrm{Cl}$ were $564(95 \% \mathrm{Cl}, \pm 1106)$ and 2.42 steps $(95 \% \mathrm{Cl}, \pm 4.74)$, respectively, for 2 consecutive 7-day recordings. The week-to-week SDD was 1564 for total steps and 6.70 for peak activity. The 7-day mean for total step activity was 5355 for the patients with hematologic malignancies and 6364 for healthy subjects $(P<0.05)$. Conclusions The results of this study indicate that there is good relative reliability for the assessment of 2 consecutive 7-day recordings of ambulatory walking activity, and it showed that the SDD derived from this sample may be useful in detecting changes in daily walking activity in hematologic cancer patients who are recovering from intensive medical treatment. The study also documented compromised levels of ambulatory walking activity among hematologic cancer patients recovering from highdose chemotherapy as compared with healthy controls.

Key words Hematologic neoplasms; Physical activity; Rehabilitation; Technology assessment; Walking. 


\section{Introduction}

The intensive medical treatment of patients with hematologic malignancies [17] is associated with numerous long-term adverse effects, including anemia, fatigue, and reduced physical exercise capacity. [8] Fatigue may compromise general activity, work, enjoyment of life, mood, walking, and relationships with others even for a length of time after medical treatment [9-11] is completed.

It has been shown that patients with hematologic malignancies may benefit from physical exercise programs in terms of maintenance or even improvement in fatigue, physical activity, and fitness levels. [12-14] A major component of daily physical activity and the most common form of exercise is walking. [15] Walking is self regulated in intensity, duration, and frequency and can be an important indicator of a person's health and fitness status.[16] The improvement of functional status is a primary goal in the rehabilitation of cancer patients. [17,18] It is thus important to be able to document quantitatively the walking activity of patients who are recovering from intensive medical treatment. An understanding of the quantity (or lack) of walking activity seems particularly important in addressing the needs of cancer patients who are recovering from intensive medical treatment.

Daily walking activity can be assessed by means of self-report diaries, questionnaires, or objective performance indicators. Self-report measures of walking activity have been reported to be imprecise compared with objective measures. $[19,20]$ Relatively new techniques allowing unobtrusive long-term activity monitoring with the use of pedometers and microprocessor-based accelerometer recorders may provide a clearer view of how much an individual actually walks in his/her own surroundings. [21] However, the major limitations of many pedometers are their accuracy and reliability $[21,22]$ because they are worn at the waist and thus are sensitive to vertical movement. Therefore, the response may be affected by position, mode of the pedometer attachment, movement style, and walking speed of the individual being monitored. [21,22] Microprocessor-based accelerometers are developed to overcome the limitations of waist-attached devices because settings can be adapted according to height, cadence, and the walking style of the subject. [22]

The accuracy of these devices has consistently been reported to be above 98\%. [23] Most studies of the test-retest reliability of walking activity have reported only relative reliability, such as the ICCs (varying from 0.84 to 0.97). [19-21,23-28] These statistics indicate the degree of association between 2 or more measures, [29] but they do not provide clinical guidance for assessing real changes. [30,31] Absolute reliability reflects the magnitude of the differences between 2 measures. [32] Examples of these statistics are the SE of measurement procedure and the SDD. [33,34] to be clinically useful, an assessment procedure must have a small measurement error to detect a real change. A test-retest difference in a patient with a value smaller than the SE of measurement is likely to be the result of measurement noise and is unlikely to be detected reliably in practice; a difference greater than the smallest real difference is highly likely (with $95 \%$ confidence) to be a real difference. [35] To date, there is no empiric evidence to guide the clinical assessment of real changes in long-term ambulatory walking activity in 
hematologic cancer patients who are recovering from intensive medical treatment in their home environment.

Additionally, to date, no study has attempted to quantitatively document the long-term, ambulatory walking activity of hematologic cancer patients after treatment compared with the ambulatory walking activity of healthy subjects. Among healthy individuals, 10,000 steps daily has been estimated to be of value in maintaining desired health benefits. [36] However, preliminary evidence suggests that a goal of 10,000 steps daily may not be sustainable for some groups, including older adults and those living with chronic diseases. $[28,36]$ This finding is supported by the results of a recently published metaanalysis by Bohannon [37] that provided an estimate of the number of daily steps taken by adults. The results of this meta-analysis suggested that the general number of daily steps taken was less than the recommended 10,000 steps a day and that walking activity was especially low in adults 65 years of age or older. In 1 study, [21] the mean 7-day step counts of healthy subjects (6929 steps; range, 4347-10,002 steps) was significantly greater $(P<0.001)$ than that of patients with multiple sclerosis (2985 steps; range, 689 - 5340 steps), Parkinson disease (3818 steps; range, 1611-5391 steps), or muscular disorders (3003 steps; range, 716 - 5302 steps). The purpose of this study was to determine the relative and absolute reliability of the assessment of ambulatory walking activity during 2 consecutive weeks in patients with hematologic malignancies recovering at home from their medical treatment and to compare the physical activity level of hematologic cancer patients after high-dose chemotherapy to that of healthy subjects.

\section{Methods}

\section{Participants}

Patients with a diagnosis of hematologic cancer who had completed treatment with high-dose chemotherapy were selected to participate in the study from the Departments of Oncology and Hematology of the University Hospital Zurich. Patients were excluded from the study if they were experiencing the direct side effects of high-dose chemotherapy (eg, fever, hemoglobin level $<10 \mathrm{~g} / \mathrm{dL}$, emesis, dyspnea, $\leq 36$ of 52 points on the FACT-F subscale [38]) in case of gait abnormalities, known impairment of the lower limbs, severe GVHD except for grade 1 not requiring treatment, painful joints, instable osteolyses of the vertebrae, chronic low back pain, lesions of the central or peripheral nervous system, uncontrolled cardiovascular disease, thyroid disease, or diabetes. The patients' walking activity levels were compared with those of 30 subjects without known physical impairments, injuries, or other health-related conditions that hampered walking activity. An inverse correlation has been reported between walking activity, higher BMI, [39] and increased age. [40] Therefore, the healthy subjects were matched for age $($ years \pm 5$)$, sex, and BMI $\left(B M I \pm 3 \mathrm{~kg} / \mathrm{m}^{2}\right)$ (table 1) with the patients. The healthy subjects were recruited, at random, from the hospital's employees, including laboratory assistants $(n=6)$, nurses $(n=5)$, physical therapists $(n=5)$, administrative personnel $(n=6)$, physicians $(n=1)$, researchers $(n=4)$, dieticians $(n=1)$, and technicians $(n=2)$. All participants provided written informed 
consent. The study was approved by the Ethic Commission of the Canton of Zurich.

\section{Descriptive measures}

Height was assessed to the nearest $0.5 \mathrm{~cm}$ with a wall fixed tape measure and weight to the nearest $0.5 \mathrm{~kg}$ (SECA weighting machine, Model 791a). Hemoglobin values $(\mathrm{g} / \mathrm{dL})$ were determined at the time of an outpatient visit, and self-reported fatigue was assessed with the German language version of the 13-item FACT-F Version 4. [41] This scale includes items relating to both the symptoms and consequences of fatigue and has testretest reliability $(r=0.90)$. [41] Hemoglobin values and self-reported fatigue were measured because both factors (ie, low hemoglobin levels and high fatigue levels) can have adverse effects on physical performance over time. [42]

\section{Step activity monitoring}

Step activity levels were assessed with the SAM3. ${ }^{b}$ The SAM3 measures $75 x$ $50 \times 20 \mathrm{~mm}$ and weighs approximately $38 \mathrm{~g}$. Settings of the SAM3 can be adapted according to the height, cadence, and walking style of the individual. [16] The SAM3 does not allow adjustment and does not require maintenance by the user. The SAM3 is worn on the right lateral or the left medial malleolus. It is contoured to fit comfortably against the leg (figure 1). An elastic attachment strap ensures that the monitor remains securely attached to the ankle without irritating the skin. The SAM3 continuously records the number of steps per time interval. The device is programmed and downloaded onto a host computer via a universal serial bus docking station. Programming and downloading are performed with the StepWatch Analysis Software. ${ }^{b}$ Information that was routinely recorded in the standard programming mode included the following individual gait characteristics: cadence, speed, the total length of time of the data collection (7 days), and individual identifying information. The device was calibrated by programming estimated values for walking cadence and speed based on the subject's height (patient's or healthy subject's) into the computer before providing the SAM3 to the subject. The subject was then instructed to walk at a normal preferred speed for $20 \mathrm{~m}$. The steps of the right leg were counted manually by an observer and compared with those of the SAM3. This procedure was repeated if there was a difference of 2 steps or more between the manual count and that of the step watch monitor. If the manual counting was lower than that of the step monitor, the programmer decreased the value of the cadence of the SAM3. If the manual count remained too high, the sensitivity of the SAM3 was decreased by the programmer. [16]

\section{Recording procedures of the SAM3}

Patients and healthy controls were instructed to wear the SAM3 for 7 consecutive days, excluding sleep time. Activity monitoring over a 7-day period has previously been found to result in reliable and representative measures of an individual's movements on a day-to-day basis. [21] All participants were instructed to leave the SAM3 on the ankle if they had to rest or lay down during the day and to perform their daily activities as usual. After 7 days, the participants returned to the hospital, where the data were 
downloaded into a database. For patients, this procedure was repeated for a second consecutive 7-day period. When returning the step monitor, we asked the patients if they changed their walking behavior because of health related factors during the 2-week recording time. The walking activity of healthy controls was measured for the initial consecutive 7-day period only.

\section{SAM3 parameters}

The parameters assessed by the SAM3 for this study were (1) total steps a day and (2) the peak activity index (the maximum number of steps recorded per time interval for each day).

\section{Statistical analyses}

Normality of the data was tested with the Kolmogorov-Smirnov test. [43] Data were analyzed by using the ICC3.1, with 95\% CI. [29] An ICC greater than 0.75 was defined as high reliability. [29]

The SE of measurement procedure was calculated from the average known SD and the relative reliability coefficient $(r)$ of the measurement procedure used for our sample: SE of measurement procedure $=\operatorname{SD}(\sqrt{ } 1-r)$. $[29,34,44,45]$ The corresponding $95 \% \mathrm{Cl}$, in which the true score (drawn from the normally distributed population) is expected, was $\pm 1.96 \times$ SE of measurement procedure. [46-48] The broader the limits of the $95 \% \mathrm{Cl}$, the less confident the estimation of the true score and, as a consequence, the less confident the detection of the real change because of intervention. [45] This knowledge about the SE of measurement procedure is necessary before one can say that such a change has occurred. [46,49] Moreover, when analyzing a difference between 2 consecutive observations, one must consider the SE of measurement procedure of the observed score for both the first week of walking activity (SE of measurement procedure [first week]) and the second-week (SE of measurement procedure [second week]) observations. The SDD is known as the measure of statistically significant change between 2 independently obtained measurements. Given a probability value of alpha equal to 0.05 as indication for statistical significance, the SDD is estimated as $1.96 \times$ (SE of measurement procedure [first week] ${ }^{2}+\mathrm{SE}$ of measurement procedure [second week] $^{2}$ ). [31] Assuming that the SE of measurement procedure of the observed score of the first and second observations is equal, the SDD is $1.96 \times \sqrt{2} \times \mathrm{SE}$ of measurement procedure. For a statistically significant change between 2 separate observations to be detected, this change must be at least the SDD of the measurement procedure. [34]

We determined CV (SD/mean x 100\%) to examine the individual dayto-day and week-to-week within-subject variability for walking activity (table 2) in hematologic cancer patients. [29] Differences between patients' means for total steps and peak activity were calculated with a paired Student $t$ test to quantify the systematic error (also known as bias) of the step activity monitor. Differences between the patients' and the healthy participants' means for total steps and peak activity were determined with an independent sample $t$ test. [29] All statistical analyses were performed by using SPSS 12.0.1 for Windows ${ }^{c}$ and Excel 2003 for Windows. ${ }^{d}$

\section{Results}


Forty-nine adult patients with a diagnosis of hematologic cancer who had completed treatment with high-dose chemotherapy were selected. Five patients were excluded because of low hemoglobin values and/or severe fatigue, and 14 patients were not interested in participating. Specifically, the study sample $(n=30)$ consisted of 14 leukemia patients treated with induction chemotherapy after peripheral blood stem cell transplantation, 11 nonHodgkin lymphoma patients treated with high-dose chemotherapy alone ( $\mathrm{n}$ $=10)$ or high-dose chemotherapy after autologous stem cell transplantation $(n=1)$, and 4 multiple myeloma/plasmacytoma patients treated with high-dose chemotherapy alone $(n=3)$ or high-dose chemotherapy after 2 cycles of autologous stem cell transplantation $(n=1)$. All patients participating were in a physically stable condition. Seven of the 30 patients (23\%) did not wear the SAM3 consistently during the 7-day period (during waking hours) and were, for that reason, excluded from the analyses. None of the patients reported change in walking behavior because of health-related factors. Thus, the final analysis was performed in 23 patients (12 women/11 men) (see table 1). The accuracy of the SAM 3 was $98 \%$ in this study based on a comparison of the number of steps counted by the SAM3 with the observed steps after the cadence had been identified. All results of the step activity measurements and the difference for step activity measurements between week 1 and week 2 were normally distributed.

The ICCs including the $95 \% \mathrm{Cl}$ for total steps and peak activity were $0.90(95 \% \mathrm{Cl}, 0.75-0.98)$ and $0.85(95 \% \mathrm{Cl}, 0.66-0.94)$, respectively. The absolute reliability for total steps and peak activity expressed as the SE of measurement procedure including the $95 \% \mathrm{Cl}$ and the SDD was $564(95 \%$ $\pm 1106)$ and $2.42(95 \% \mathrm{Cl} \pm 4.74)$, respectively. The SDD was 1564 for total steps and 6.7 for peak activity. The 7-day measurement period (week 1) revealed a day-to-day variability for total steps in hematologic cancer patients as follows: a range of 14,903 (minimum 1008, maximum 15,911), a mean \pm SD of $5355 \pm 2678$. The day-to-day and week-to-week mean CV for step counts was $35.17 \%$ and $13.23 \%$, respectively (see table 2). The day-to-day and week-to-week mean CV for peak activity was $18.61 \%$ and $6.90 \%$, respectively. There were no significant changes for the total steps and peak activity parameters (table 3 ) or were there significant changes for the patient group in hemoglobin values or self-reported fatigue between weeks 1 and 2 . The patient group yielded significantly lower mean values for the parameters "total steps" and "peak activity index" than the healthy comparison group (table 4).

\section{Discussion}

This is the first study that provides clinical guidance for the assessment of real changes in long-term day-to-day walking in patients with hematologic malignancies after medical treatment. We have used the ICC (with accompanying $95 \% \mathrm{Cl}$ ) and the CV to estimate relative reliability and the SE of measurement procedure (with accompanying $95 \% \mathrm{Cl}$ ) and the SDD to estimate measurement error. To be of practical use, the results should be interpreted as follows: the measurements exhibited good relative reliability with an ICC value of $0.9(95 \% \mathrm{Cl}, 0.75-0.98)$, and a CV of $13.23 \%$ for the variable total steps. 
For the variable peak activity, we estimated an ICC of $0.85(95 \% \mathrm{Cl}, 0.66$ 0.94 ) and a CV of $6.9 \%$. Although patients reported that they did not change their walking behavior because of changes in health status or symptom burden during the 2-week assessment period, the absolute reliability or measurement error of this parameter is affected by the relatively large variance statistics of the measurements total steps week 1 and week 2, which were 3,719,690 and 2,695,540, respectively (calculated as the square of the SD week 1 [1928.65] and week 2 [1641.81]). The variance statistics of the variable peak activity were 44.89 for week 1 and 42.25 for week 2 . When taking the measurement error into account (SE of the measure procedure $=$ 564 for total steps, 2.42 for peak activity), an SDD greater than 1564 total steps (or 6.7 for peak activity) between 2 consecutive 7-day measures should be interpreted as the real change in patients' total steps daily activity. It is reasonable to assume that a significant increase in both variables after, for example, participation in a moderate walking exercise program can be measured with the SAM3. Conversely, however, it would not be possible to detect a real change in daily total step activity or peak activity if the measurement error is larger than the improvement or deterioration in the walking activity of the patient.

Although the SAM3 is a lightweight and unobtrusive device, it is remarkable that $23 \%$ of the patients did not comply with wearing the step monitor consistently during the 2 consecutive weeks. To gather representative data, clinicians and researchers should emphasize the importance of the measurement protocol to the participant when wearing a step monitor device. However, the original data for total step activity in 30 patients with hematologic malignancies yielded an equal reliability result for the ICC (0.90). The SDD was 2126. The variance statistics for week 1 and week 2 in 30 patients were $3,342,063$ and 3,117,484, respectively. The ICC for peak activity in the original 30 patients was 0.88 and the SDD 8.98. The variance statistic for week 1 and week 2 in 30 patients was 49.28 . For the correctness of analysis, we chose to present the data of the 23 complying patients. We compared the total daily ambulatory step activity in patients with hematologic malignancies with those of age-, sex-, and BMI-matched healthy participants. The healthy subjects performed significantly more total daily steps and also had a higher peak activity index, which indicated that the healthy subjects walked with an overall higher intensity than the cancer patients (see table 4). With the improvement of prognosis in hematologic malignancies, walking activity may become an important component in continuing care programs (eg, for physical activity programs that emphasize walking activity and that also consider the intensity of the performed steps as important). Therefore, the amount of steps a day and the peak activity index both appear to be of particular relevance in the case of patients with hematologic cancer who are recovering from systemic treatment because they seem especially suited as outcome parameters for intervention studies.

The findings of our cross-sectional investigation with regard to daily walking activity are supported by several earlier studies. Keats et al. [50] reported that physical activity significantly declined in adolescents who were treated for leukemia and did not return to pretreatment levels after the completion of treatment. Florin et al. [51] reported that long-term survivors of childhoodleukemia were less likely to meet physical activity recommendations 
and that lowered levels of physical activity increase the risk of cardiovascular disease, osteoporosis, and all-cause mortality. Thus, the long-term implications of a reduction in physical activity, including daily walking activity, may be far reaching for patients with hematologic malignancies. However, longitudinal studies are needed to document the changes in physical activity level in this population of patients.

Our reliability results are similar to those observed in other studies. A test-retest coefficient of 0.97 was observed in 1 study [19] in which the stride counts were counted during a 6-minute walking test. Resnick et al. [24] found an ICC value of 0.84 in a test-retest design when comparing 1-minute walks in elderly adults. Haeuber et al. [25] reported a reliability of 0.96 when stride counts were assessed in a 2- $x$ 24-hour test-retest situation. Finally, Busse et al. [21] reported an ICC of 0.86 and 0.89 in neurologic patients and healthy persons, respectively, in a $2-x$ 7-day test-retest design in which daily steps were counted. In this study, the day-to-day variability in both healthy individuals and those with neurologic impairment was relatively high as reflected by the CV. [21] The average CV for day-to-day step counts in healthy subjects was $28 \%$, and, in week measures in healthy subjects was $8.8 \%$, and, in neurologic patients, it was $12 \%$. [21] The healthy subjects (mean age, 47y) in our study walked 6363 steps during a consecutive 7-day period. This performance is comparable with the study of Busse et al,21 in which healthy subjects (mean age, 43y) were reported to walk 6520 steps daily. On the other hand, a meta-analyses of Bohannon [37] provided a daily step count average of 9448 steps (95\% Cl, 8899 - 9996) taken by healthy adults. The average daily step count performance was divided into 2 groups: subjects younger than 65 years of age who performed 9797 (95\% Cl, 9216 $10,377)$ steps daily and adults over 65 years of age who performed on average $6565(95 \% \mathrm{Cl}, 4897$ - 8233) daily steps. As a result, the participating hematologic patients and healthy subjects in our study would be placed in the low active (5000 - 7499 steps/day) category for walking activity [36,37] together with the adults over 65 years of age from the meta-analyses of Bohannon. [37]

We would like to note that our results should be interpreted with some caution. First, measures of unconstrained walking activity directly assess the real-life behavior of the study participants who are facing challenges when walking at home or in the community. [28] During the measurements, external conditions such as weather, [52] suitability of walking activities, or mediators of walking activities such as self-efficacy, social support, or decisional balance [53] and the health condition of the patients may influence the variation observed in walking activity. However, in test situations such as ours, it is possible that the participants, both the cancer patients and the healthy controls, are motivated to perform more walking activity, despite explicit instructions to perform their daily activities as usual and not to perform extra physical activity. Yet, it might also be possible that the participating patients in our study were healthier than other hematologic cancer patients at this stage of their recovery. The main reason for 11 patients not to participate was that they felt too fatigued or too weak. To more fully investigate the multiple sources of potential error in assessing daily walking activity, future work should address a broader set of reproducibility indicators. In line with the generalizability theory, multiple sources of measurement error in walking 
activity to be evaluated include whether patients consistently wear the SAM3 (ie, adherence), the between- and within subject variation in day-to-day walking behavior as a function of the number of hours the SAM3 was worn, evidence that the time periods in which walking activity was measured were typical, and the possible effect of health-related factors such as fatigue, infection, and so on on day-to-day walking activity. Using this generalizability approach, reproducibility can be assessed and tailored to the intended applications of the SAM3 in cancer patients. [54]

Second, the point in time at which the patient assessments took place varied considerably (see table 1), and, therefore, some patients may have had the possibility to recover more from the side effects of high-dose chemotherapy than others. This may have influenced the variation observed in the day to-day walking activity. However, there are several reasons why we believe that the chosen sample of hematologic cancer patients after medical treatment was adequate. Our participants were recruited on average 5 months after medical treatment. None of the patients were in the acute phase or experienced the direct side effects of medical treatment. Furthermore, the haemoglobin values and the self-reported symptoms of fatigue did not change significantly over the measurement period and were therefore indicative for a stable physical condition. Nevertheless, one cannot rule out the possibility of a changing physical health status during the 2-week period in which the patients were observed.

Third, the cancer patients in our study were compared with a relatively select sample of healthy participants working in a university hospital setting. As compared with the meta-analyses of Bohannon, [37] other results might have emerged if the comparison had been made with individuals without known physical impairments, injuries, or health-related conditions that would influence walking behavior working in a wider range of settings, including individuals who were either not working or had other societal functions (eg, students, homemakers, and so on). Despite these caveats, we believe that our study provides useful first data regarding the relative reliability of and measurement error associated with walking activity as measured with the SAM3 when used with hematologic cancer patients and documents the sustained deficit in walking activity levels experienced by these patients when compared with age- and sex-matched controls.

\section{Conclusions}

This study represents an ongoing process toward developing accurate and reliable tools to describe the individual walking performance in the patients' own environment. The results of this study indicate that there is good relative reliability for the assessment of 2 consecutive 7-day ambulatory walking activity in hematologic cancer patients. This study showed that the SDD derived from this sample may be useful in detecting changes in daily walking activity in hematologic cancer patients who are recovering from intensive medical treatment. The study also documented compromised levels of ambulatory walking activity among hematologic cancer patients recovering from high-dose chemotherapy as compared with healthy controls.

\section{Acknowledgments}


We thank Pamela Hofer, MSc, and Michèle Hubli, MSc, for instruction in the use of the SAM3 and for data management and Leanne Pobjoy for her assistance with the English editorial service. We also thank all of the physicians from the Department of Medical Oncology and from the Department of Hematology, University Hospital of Zurich, for referring patients to this study.

\section{References}

1. Koistinen P, Räty R, Itälä $M$, et al.: Finnish Leukaemia Group. Long-term outcome of intensive chemotherapy for adults with de novo acute myeloid leukaemia (AML): the nationwide AML-92 study by the Finnish Leukaemia Group. Eur J Haematol 78: 477-486, 2007

2. Lacy MQ, Gertz MA, Dispenzieri $A$, et al.: Long-term results of response to therapy, time to progression, and survival with lenalidomide plus dexamethasone in newly diagnosed myeloma. Mayo Clin Proc 82: 1179-1184, 2007

3. Okamura J, Uike N, Utsunomiya A, et al.: Allogeneic stem cell transplantation for adult T-cell leukemia/lymphoma. Int J Hematol 86: 118125, 2007

4. Toze C, Barnett MJ: Allogeneic haemopoietic stem cell transplantation for non-Hodgkin's lymphoma. Best Pract Res Clin Haematol 15: 481-504, 2002

5. Axdorph U, Stenke L, Grimfors G, et al., for the Swedish CML Group. Intensive chemotherapy in patients with chronic myelogenous leukaemia $(\mathrm{CML})$ in accelerated or blastic phase-a report from the Swedish CML Group. Br J Haematol 118: 1048-1054, 2002

6. Estey E, Döhner H: Acute myeloid leukaemia. Lancet 368: 1894-1907, 2006

7. Koreth J, Cutler CS, Djulbegovic B, et al.: High-dose therapy with single autologous transplantation versus chemotherapy for newly diagnosed multiple myeloma: a systematic review and meta-analysis of randomized controlled trials. Biol Blood Marrow Transplant 13:183-196, 2007

8. Evans WJ, Lambert C: Physiological basis of fatigue. Am J Phys Med Rehabil 86(1 Suppl):29-46, 2007

9. Wang XS, Giralt SA, Mendoza TR, et al.: Clinical factors associated with cancer-related fatigue in patients being treated for leukemia and nonHodgkin's lymphoma. J Clin Oncol 20: 1319-1328, 2002

10. Hjermstad MJ, Evensen SA, Kvaløy SO, et al.: Health-related quality of life 1 year after allogeneic or autologous stem-cell transplantation: a prospective study. J Clin Oncol 17:706-718, 1999

11. Hjermstad MJ, Knobel H, Brinch L, et al.: A prospective study of healthrelated quality of life, fatigue, anxiety and depression 3-5 years after stem cell transplantation. Bone Marrow Transplant 34: 257-266, 2004

12. Dimeo F.: Exercise for cancer patients: a new challenge in sports medicine. Br J Sports Med 34:160-161, 2000

13. Dimeo F, Schmittel A, Fietz T, et al.: Physical performance, depression, immune status and fatigue in patients with haematological malignancies after treatment. Ann Oncol 15:1237-1242, 2004

14. Hayes SC, Davies PS, Parker TW, et al.: Role of a mixed type, moderate intensity exercise programme after peripheral blood stem cell transplantation. Br J Sports Med 38: 304-309, 2004 
15. Manson JE, Greenland P, LaCroix AZ, et al. : Walking compared with vigorous exercise for the prevention of cardiovascular events in women. $\mathrm{N}$ Engl J Med 347: 716-725, 2002

16. McDonald CM, Widman L, Abresch RT, et al.: Utility of a step activity monitor for the measurement of daily ambulatory activity in children. Arch Phys Med Rehabil 86: 793-801, 2005

17. Brown JK, Byers T, Doyle C, et al.: Nutrition and physical activity during and after cancer treatment: an American Cancer Society guide for informed choices. CA Cancer J Clin 53:268-291, 2003

18. Knols R, Aaronson NK, Uebelhart D, et al. : Physical exercise in cancer patients during and after medical treatment: a systematic review of randomized and controlled clinical trials. J Clin Oncol 23: 3830-3842, 2005

19. Macko RF, Haeuber E, Shaughnessy M, et al.: Microprocessor based ambulatory activity monitoring in stroke patients. Med Sci Sports Exerc 34: 394-399, 2002

20. Smith DG, Domholdt E, Coleman KL, et al.: Ambulatory activity in men with diabetes: Relationship between self-reported and real-world performance-based measures. J Rehabil Res Dev 41: 571-580, 2004

21. Busse ME, Pearson OR, Van Deursen R, et al. : Quantified measurement of activity provides insight into motor function and recovery in neurological disease. J Neurol Neurosurg Psychiatry 75: 884-88, 2004

22. Shepherd EF, Toloza E, McClung CD, et al.: Step activity monitor: increased accuracy in quantifying ambulatory activity. J Orthop Res 17:703708, 1999

23. Coleman KL, Smith DG, Boone DA, et al.: Step activity monitor: long-term, continuous recording of ambulatory function. J Rehabil Res Dev 36: 8-18, 1999

24. Resnick B, Nahm ES, Orwig D, et al.: Measurement of activity in older adults: reliability and validity of the Step Activity Monitor. J Nurs Meas 9: 275290, 2001

25. Haeuber E, Shaughnessy M, Forrester LW, et al.: Accelerometer monitoring of home- and community-based ambulatory activity after stroke. Arch Phys Med Rehabil 85: 1997-2001, 2004

26. Busse ME, Wiles CM, van Deursen RM: Community walking activity in neurological disorders with leg weakness. J Neurol Neurosurg Psychiatry 77: 359-362, 2006

27. Song KM, Bjornson KF, Cappello T, et al.: Use of the StepWatch activity monitor for characterization of normal activity levels of children. J Pediatr Orthop 26: 245-249, 2006

28. Cavanaugh JT, Coleman KL, Gaines JM, et al.: Using step activity monitoring to characterize ambulatory activity in community-dwelling older adults. J Am Geriatr Soc 55:120-124, 2007

29. Portney L, Watkins M: Foundations of clinical research. Applications to practice. 1st ed. Norwalk: Appleton and Lang; 1993.

30. de Vet HC, Terwee CB, Knol DL, et al.: When to use agreement versus reliability measures. J Clin Epidemiol 59:1033-1039, 2006

31. Guyatt G, Walter S, Norman G: Measuring change over time: assessing the usefulness of evaluative instruments. J Chronic Dis 40: 171-178, 1987 
32. Liaw LJ, Hsieh CL, Lo SK, et al.: The relative and absolute reliability of two balance performance measures in chronic stroke patients. Disabil Rehabil 30: 656-661, 2008

33. Bowden MG, Behrman AB: Step activity monitor and test re-test reliability in persons with incomplete spinal cord injury. J Rehabil Res Dev 44: 355-362, 2007

34. Kropmans TH, Dijkstra PU, Stegenga B, et al.: Smallest detectable difference in outcome variables related to painful restriction of the temporomandibular joint. J Dent Res 78: 784-789, 1999

35. Sole G, Hamrén J, Milosavljevic S, et al.: Test-retest reliability of isokinetic knee extension and flexion. Arch Phys Med Rehabil 88: 626-631, 2008

36. Tudor-Locke C, Bassett DR Jr.: How many steps/day are enough? Preliminary pedometer indices for public health. Sports Med 34: 1-8, 2004

37. Bohannon RW: Number of pedometer-assessed steps taken per day by adults: a descriptive meta-analyses. Phys Ther 87: 1642-1650, 2007

38. Palumbo A, Petrucci MT, Lauta VM, et al.: Correlation between fatigue and hemoglobin level in multiple myeloma patients: results of a crosssectional study. Haematologica 90: 858-860, 2005

39. Chan CB, Spangler E, Valcour J, et al.: Cross-sectional relationship of pedometer-determined ambulatory activity to indicators of health. Obes Res 11: 1563-1570, 2003

40. Toupin JM, Dujardin F: Quantitative evaluation of indoor walking activities related to age. Eur J Orthop Surg Traumatol 9: 27-30, 1999

41. Cella D: The Functional Assessment of Cancer Therapy-Anemia (FACTAn) Scale: a new tool for the assessment of outcomes in cancer anemia and fatigue. Semin Hematol 34(3 Suppl 2): 13-19, 1997

42. Birgegård G, Gascón P, Ludwig H: Evaluation of anaemia in patients with multiple myeloma and lymphoma: findings of the European CANCER ANAEMIA SURVEY. Eur J Haematol 77: 378-386, 2006

43. Siegel S, Castellan N: Non-parametric statistics for the behavioural sciences. 1st ed. Norwalk-New York: Mcgraw-Hill; 1988.

44. Cronbach JL, Gleser GC, Nanda H, et al.: The dependability of behavioral measurements: theory of generizibility for scores and profiles. New York: John Wiley and Sons; 1972.

45. Ottenbacher KJ, Johnson MB, Hojem M: The significance of clinical change and clinical change of significance: issues and methods. Am $\mathrm{J}$ Occupat Ther 42: 156-162, 1988

46. Roebroeck ME, Harlaar J, Lankhorst GL: Reliability assessment of isometric knee extension measurements with a computerassisted hand-held dynamometer. Arch Phys Med Rehabil 79: 442-448, 1998

47. IJzerman MJ, Baardman G, Van 't Hof MA, et al.: Validity and reproducibility of crutch force and hearth rate measurements to assess energy expenditure of paraplegic gait. Arch Phys Med Rehabil 80: 1017-1023, 1999

48. de Bruin ED, Rozendal RH, Stüssi E: Reliability of phase-velocity measurements of tibial bone. Phys Ther 78: 1166-1174, 1998

49. Hayes KW: The effect of awareness of measurement error on physical therapists confidence in their decisions. Phys Ther 72: 515-525, 1992

50. Keats MR, Culos-Reed SN, Courneya KS, et al.: An examination of physical activity behaviors in a sample of adolescent cancer survivors. J Pediatr Oncol Nurs 23: 135-142, 2006 
51. Florin TA, Fryer GE, Miyoshi T, et al.: Physical inactivity in adult survivors of childhood acute lymphoblastic leukemia: a report from the childhood cancer survivor study. Cancer Epidemiol Biomarkers Prev 16: 1356-1363, 2007

52. Frank JS, Patla AE: Balance and mobility challenges in older adults: implications for preserving community. Am J Prev Med 25 (3 Suppl 2): 157163, 2003

53. Marcus B, Forsyth L: Motivating people to be physically active. 1st ed. Champaign: Human Kinetics; 2003.

54. Roebroeck ME, Harlaar J, Lankhorst GJ: The application of generalizability theory to reliability assessment: an illustration using isometric force measurements. Phys Ther 73: 386-389, 1999

\section{Suppliers}

a. Hess Medizintechnik AG, Grabenstrasse 2, 8865 Bilten, Switzerland.

b. Cymatech Corp, 8515 35th Ave NE, Ste C, Seattle, WA 98115.

c. SPSS Inc. (Switzerland), Schneckenmannstrasse 258044 Zurich, Switzerland.

d. Microsoft Inc. (Switzerland), Richtistrasse 3, 8304 Wallisellen (Zurich), Switzerland.

Abbreviations: $\mathrm{BMI}$; body mass index, $\mathrm{Cl}$; confidence interval, $\mathrm{CV}$; coefficient of variation, FACT-F; Functional Assessment of Cancer Therapy-Fatigue, GVHD; graft-versus-host disease, ICC; intraclass correlation coefficient, SDD; smallest detectable difference, SAM3; StepWatch Activity Monitor. 


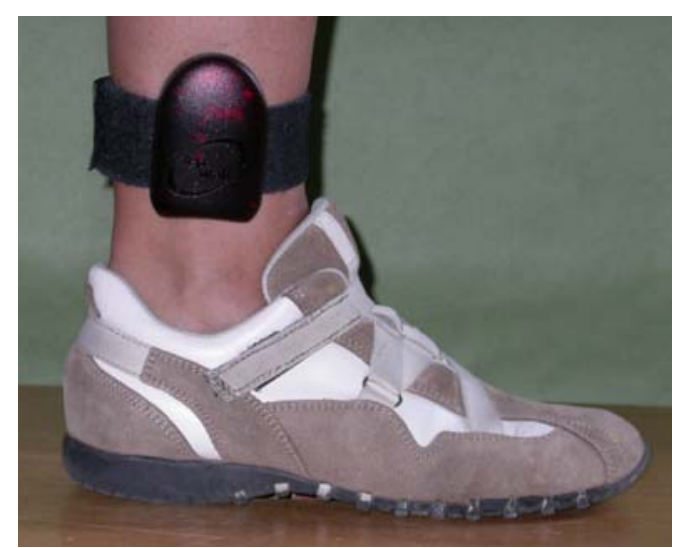

Figure 1: The StepWatch Activity Monitor $3\left(\mathrm{SAM}^{\odot}{ }^{\odot}\right.$, Cymatech Corporation, Seattle, WA, USA) is contoured to fit comfortably against the leg. An elastic attachment strap ensures that the monitor remains securely attached to the ankle without irritating the skin. 


\begin{tabular}{|c|c|c|c|}
\hline & $\begin{array}{l}\text { Patients with } \\
\text { hematological } \\
\text { malignancies }(n=23)\end{array}$ & $\begin{array}{l}\text { Healthy } \\
\text { Participants } \\
(n=30)\end{array}$ & $\mathrm{p}$-value \\
\hline Mean Age (sd) & $47.83(14.57)$ & $46.20(11.83)$ & .656 \\
\hline Mean Height in $\mathrm{cm}$ (sd) & 173.35 (9.59) & $173.47(9.09)$ & .963 \\
\hline Mean Weight in $\mathrm{kg}(\mathrm{sd})$ & 77.95 (12.90) & $72.15(17.03)$ & .377 \\
\hline Mean BMI in $\mathrm{kg} / \mathrm{m}^{2}$ (sd) & 25.08 (3.39) & $23.80(4.01)$ & .225 \\
\hline $\begin{array}{l}\text { Mean time (days) (sd) } \\
\text { since medical } \\
\text { treatment }\end{array}$ & $142.65(68.74)$ & n.a. & n.a. \\
\hline $\begin{array}{l}\text { Fatigue FACT-AN/F } \\
\text { (sd) }\end{array}$ & 40.17 (8.28) & n.a. & n.a. \\
\hline Hemoglobin $(g / d l)(s d)$ & $12.25(1.53)$ & n.a. & n.a. \\
\hline ean valu & iatoloaic & ncies and he & \\
\hline
\end{tabular}




\begin{tabular}{|c|c|c|c|c|c|c|c|c|c|c|c|c|c|c|}
\hline \multicolumn{8}{|c|}{ Step count } & \multicolumn{2}{|c|}{$\begin{array}{c}7 \text { day mean } \\
\text { step count }\end{array}$} & \multicolumn{2}{|c|}{$\begin{array}{l}\text { Day-to-day } \\
\text { step count }\end{array}$} & \multicolumn{3}{|c|}{$\begin{array}{c}\text { Week-to-week } \\
\text { mean step count }\end{array}$} \\
\hline Patient & Day 1 & Day 2 & Day 3 & Day 4 & Day 5 & Day 6 & Day7 & Wk 1 & Wk 2 & SD & CV \% & Mean & SD & CV \% \\
\hline 1 & 3191 & 4683 & 5521 & 2724 & 7073 & 5537 & 6574 & 5043 & 4125 & 1626.58 & 32,25 & 4584 & 649.43 & 14,17 \\
\hline 2 & 2105 & 5470 & 3546 & 4907 & 3673 & 4771 & 3582 & 4008 & 3257 & 1129.63 & 28,19 & 3632 & 530.73 & 14,61 \\
\hline 3 & 5317 & 5142 & 4055 & 5506 & 4737 & 8161 & 5201 & 5446 & 4653 & 1289.80 & 23,69 & 5049 & 560.23 & 11,09 \\
\hline 4 & 1766 & 6958 & 3492 & 4770 & 4582 & 4176 & 4439 & 4312 & 3599 & 1553.12 & 36,02 & 3956 & 503.86 & 12,74 \\
\hline 5 & 1467 & 2100 & 3386 & 7791 & 7579 & 8677 & 5175 & 5168 & 6224 & 2923.80 & 56,58 & 5696 & 746.60 & 13,11 \\
\hline 6 & 4519 & 5284 & 5742 & 4853 & 2916 & 4172 & 4106 & 4513 & 3746 & 917.68 & 20,33 & 4130 & 542.25 & 13,13 \\
\hline 7 & 3598 & 15911 & 12074 & 13799 & 9845 & 9125 & 12282 & 10948 & 9484 & 3965.88 & 36,23 & 10216 & 1034.70 & 10,13 \\
\hline 8 & 4529 & 4212 & 7398 & 6272 & 5959 & 3764 & 8101 & 5748 & 6644 & 1651.25 & 28,73 & 6196 & 633.87 & 10,23 \\
\hline 9 & 5616 & 10566 & 9350 & 8588 & 5508 & 4130 & 7150 & 7273 & 4699 & 2332.43 & 32,07 & 5986 & 1819.69 & 30,40 \\
\hline 10 & 2038 & 6052 & 4786 & 3768 & 5090 & 4023 & 4979 & 4391 & 5583 & 1278.64 & 29,12 & 4987 & 843.17 & 16,91 \\
\hline 11 & 4406 & 4435 & 3821 & 4590 & 3039 & 7312 & 6839 & 4920 & 4981 & 1568.36 & 31,88 & 4951 & 42.93 & 0,87 \\
\hline 12 & 3345 & 6378 & 4737 & 6468 & 4396 & 2255 & 7531 & 5016 & 4744 & 1878.22 & 37,45 & 4880 & 192.23 & 3,94 \\
\hline 13 & 2675 & 2613 & 5033 & 4673 & 1403 & 8206 & 1822 & 3775 & 3310 & 2381.30 & 63,08 & 3543 & 328.60 & 9,28 \\
\hline 14 & 1167 & 5076 & 7599 & 2549 & 5766 & 4203 & 5641 & 4572 & 3048 & 2152.82 & 47,09 & 3810 & 1077.02 & 28,27 \\
\hline 15 & 5351 & 5998 & 8396 & 9665 & 8348 & 9771 & 7757 & 7898 & 5581 & 1693.03 & 21,44 & 6739 & 1638.67 & 24,32 \\
\hline 16 & 5785 & 6223 & 8487 & 11949 & 7867 & 8746 & 9381 & 8348 & 7952 & 2062.68 & 24,71 & 8150 & 280.01 & 3,44 \\
\hline 17 & 2529 & 7137 & 11475 & 5427 & 6915 & 4378 & 7410 & 6467 & 5583 & 2815.80 & 43,54 & 6025 & 625.08 & 10,37 \\
\hline 18 & 2635 & 3432 & 2180 & 2981 & 3163 & 2911 & 2686 & 2855 & 2324 & 404.02 & 14,15 & 2590 & 375.68 & 14,51 \\
\hline 19 & 2727 & 1319 & 2990 & 4281 & 4682 & 2222 & 1008 & 2747 & 4277 & 1384.18 & 50,39 & 3512 & 1081.97 & 30,81 \\
\hline 20 & 4509 & 7359 & 6786 & 4168 & 7220 & 5441 & 5141 & 5803 & 5682 & 1311.00 & 22,59 & 5743 & 85.96 & 1,50 \\
\hline 21 & 5790 & 3298 & 2960 & 7792 & 12884 & 6451 & 5683 & 6408 & 6028 & 3324.76 & 51,88 & 6218 & 268.80 & 4,32 \\
\hline 22 & 5326 & 7122 & 2144 & 5478 & 3169 & 2206 & 8709 & 4879 & 4695 & 2510.24 & 51,45 & 4787 & 130.41 & 2,72 \\
\hline $\begin{array}{l}23 \\
\text { Mean }\end{array}$ & 3369 & 2231 & 1348 & 3306 & 2771 & 2635 & 2813 & $\begin{array}{l}2639 \\
5356\end{array}$ & $\begin{array}{l}3683 \\
4952\end{array}$ & 690.25 & 26,16 & 3161 & 738.52 & 23,36 \\
\hline CV & & & & & & & & & & & 35.17 & & & 13.23 \\
\hline
\end{tabular}




\begin{tabular}{|c|c|c|c|c|}
\hline Total Steps / 24h & $5355.52(1928.65)$ & $\begin{array}{l}4952.39(1641.81) \\
\text { average sd wk 1+2: } 1785.23\end{array}$ & $\begin{array}{l}403.13(1051.55) \\
(-51.59 / 857.85)\end{array}$ & .080 \\
\hline Peak activity index & $43.04(6.70)$ & $\begin{array}{l}42.63(6.50) \\
\text { average sd wk 1+2: } 6.60\end{array}$ & $\begin{array}{l}0.42(5.09) \\
(-1.78 / 2.62)\end{array}$ & .698 \\
\hline
\end{tabular}


Table 4. Results of the SAM3, differences between patients with hematological malignancies and healthy participants

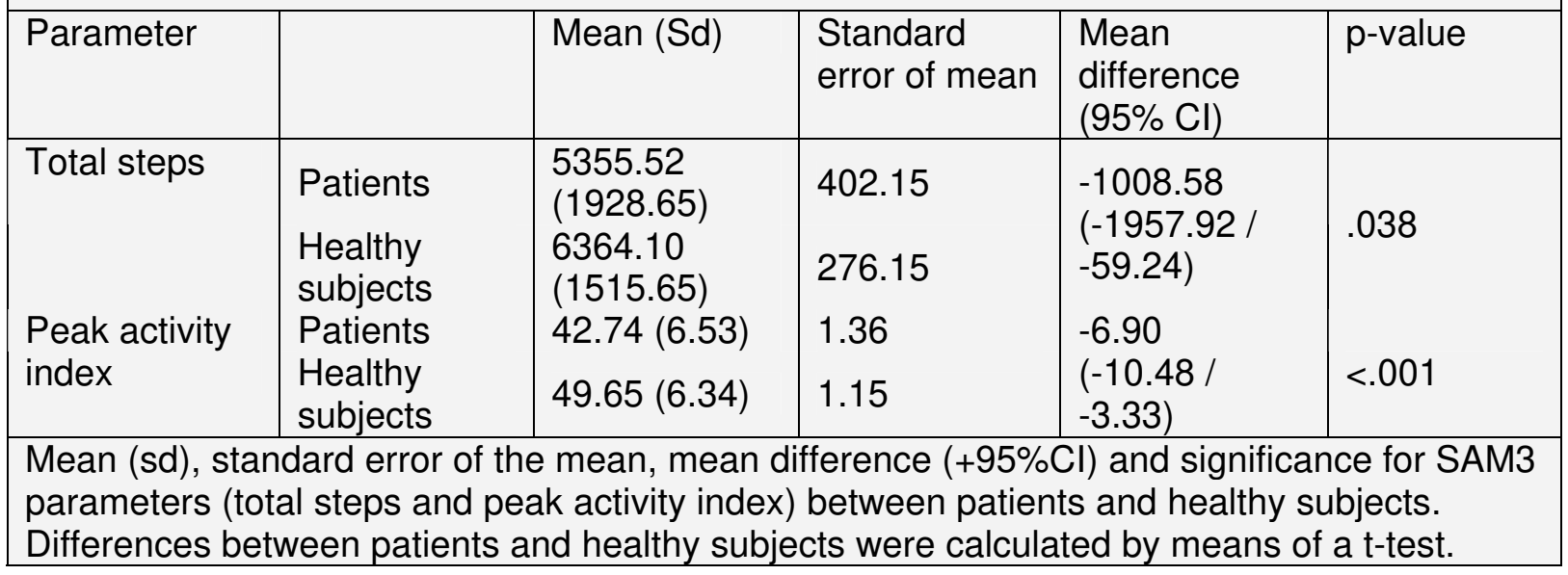

